\title{
Adaptive Tactile Control for In-hand Manipulation Tasks of Deformable Objects
}

\author{
Angel Delgado ${ }^{1, *}$, Carlos A. Jara ${ }^{2}$ and Fernando Torres ${ }^{2}$ \\ 1 University Institute of Computer Research, University of Alicante, San Vicente del Raspeig, Alicante 03690, \\ Spain; E-Mail: angel.delgado@ua.es (A.D.) \\ 2 Physics, Systems Engineering and Signal Theory Department, University of Alicante, San Vicente del \\ Raspeig, Alicante 03690, Spain; E-Mails: carlos.jara@ua.es (C.A.J.) ; fernando.torres@ua.es (F.T.) \\ * Correspondence: angel.delgado@ua.es; Tel.: +34-965-903-400 (ext. 2440); Fax : +34-965-903-464
}

\begin{abstract}
Tactile sensors are key components for a robot hand system, which are usually used to obtain the object's features. The use of tactile sensors to obtain information from the objects is an open topic of research. In this paper, a new strategy for in-hand extraction of object's properties and to control the interaction forces with robot fingers, mainly based on tactile data, is presented. The scope of this strategy is to grasp and manipulate solid objects, including rigid and soft bodies. Assuming that the hand is in an initial configuration in which the object is grasped, the properties' extraction approach is executed. After the extraction of properties is finished, the object can be classified in regard to a general body listing: rigid body, soft elastic body or soft plastic object. Once the object is classified, for in-hand manipulation tasks, the contact points between the object grasped and the fingers are maintained using the information given by the tactile sensors in order to perform manipulation tasks. Each task is defined by a sequence of basic actions, in which the contact points and applied forces are adapted depending on the action to be performed, and the estimated features for the object. The presented approach tries to imitate the behavior of human beings, in which the applied forces by the fingers are changed when the human estimates the rigidity of a body and when the fingers react to unexpected movements of the object to keep the contact points.
\end{abstract}

Keywords: tactile sensing; in-hand learning; in-hand manipulation; contact maintenance;

Acknowledgments: Research supported by Spanish Ministry of Economy, European FEDER funds, the Valencia Regional Government and University of Alicante, through projects DPI2015-68087-R, PROMETEO/2013/085 and GRE 15-05.

\section{Introduction}

The use of sensors in the robotic field is extremely necessary to give the system the capability of obtaining information about the environment that surrounds them. In the field of robotic manipulation with robot hands, two kinds of sensors are usually needed: vision and tactile sensors. Vision sensors are employed to detect the position of an object in the environment and can be used to track the movements of the system hand-object while a task is being developed. Tactile sensors are needed to detect and measure force magnitudes of contact points between hand and object. Using both together, as well as the internal information of the hand, tactile devices can be used to detect changes in the configuration hand-object.

The field of in-hand manipulation and grasping objects using robot systems is a current topic of interest in research. Many works have been developed to study the dynamics of the system, to obtain response behaviors and adaptation of the fingers when an object is manipulated. Some of these works consider that the dynamic properties of the object to be manipulated, rigid or deformable are known [1]. Many works have been presented in the literature to analyze dynamic properties, as presented in [2] where object's stiffness is analyzed using a Finite Element System. For robots which interact in real environments (domestic, industrial, services, etc.), the number of possible objects that can be grasped is very high. Robots with the capability of recognizing shapes, weights or deformation behaviors, will be more useful and versatile. Because of this, a precise sensory system is needed, 
which can obtain and use data from different sources (internal, visual and tactile). When this information is treated properly, a reliable system can interact with different kind of objects in different environments. Following this research line, many works have been developed. In [3-7] multisensory systems are used to control the manipulation of deformable bodies. The vision system is used to track the deformations on the objects. This information can be also compared with models of the objects, when the dynamic parameters are known [8]. In other works published, the manipulation control is focused on the processing of tactile data [9-11]. In all these cases, the tactile information is used to control and adapt the interaction forces that produce movements in the object.

In other type of approaches, human-inspired controllers are employed to move the fingers, as the presented in [12]. The human beings' technique treats to feel the deformation or rigidity of an object, and to adapt the movements of the fingers during a manipulation task, depending more on tactile information than visual information. This technique has been tried to reproduce in some research works $[13,14]$. When a tactile sensor is used as the main sensor device of the robotic system, the specifications of this element are key points for the reliability of the control system. A wide number of tactile sensors have been studied and used in different research works [15, 16]. Tactile data can be used to retrieve important information such as position, force magnitude and direction of the applied forces. These obtained values can be processed along with internal information of the positions of the robot to estimate more complex features of the object, such as shape, stiffness or pose [17-19]. Tactile data can also be used as a control variable, as it is used in [20]. In this last work, authors present a tactile-servo controller to adapt the movements of an articulated robot.

In this paper, a new human-inspired framework to extract objects' properties and to maintain contacts with different kind of objects is proposed. This framework is based on the information given by the internal data of a robotic hand (kinematics), and pressure information using a tactile sensor. The idea of this approach is to reproduce the behavior of a human hand when it holds an object, and the dynamic properties of the object are unknown. In contrast to model-based strategies that control the grasping and manipulation processes using a dynamic model of the objects, the authors of this paper present a model-independent strategy. Despite of losing precision in the control of the shape of the objects when deformations occur, experiments demonstrate that a reliable estimation of the objects' properties and a stable control of the contact forces can be achieved using only tactile and internal information. This novel strategy is composed by the following stages: a rigidity estimation algorithm to obtain a reliable value of how a body could be deformed, a control strategy whose goal is to maintain points of contact in the center of the area of the fingertips of the hand, and a task planner that uses the estimation of the rigidity obtained in the first stage and the kind of task to be carried out to set the needed finger configuration. The principal advantage of the proposed strategy is that it can be used in real robotic systems and with any kind of objects, because a previously given model of the object to be manipulated is not needed. In many manufacturing processes, robots should manipulate a wide variety of undefined objects, with many different mass and rigidity properties. Having a precise model for all kind of objects is frequently impossible, so the presented strategy could be a real alternative solution for many robot manipulation tasks. Besides, the independence of a model makes it possible to obtain lower processing times, a key feature needed in many manufacturing processes.

The paper is organized as follows: Section 2 describes the architecture of the robotic manipulation system. Afterwards, Section 3 and Section 4 describe the proposed techniques in which the approach of the paper is based: first, the algorithm for rigidity estimation is described in detail, then the control technique to maintain contact points is presented, and finally the task planner performance is explained. Experiments and results are shown in Section 5, which demonstrate the validity of the presented framework. Conclusions are reported in the final section.

\section{System Description}

The system is composed by a Shadow robot hand [21], and the Tekscan Grip tactile sensor [22] (see Figure 1). The sensor can be adapted to the shape of the robot hand, and it is divided in eighteen regions which are distributed over the fingers and palm. The sensor is attached to the hand, such that each region of the sensor is located in a relative area of the hand. Each of the regions is 
composed by different number of cells, which are resistive sensors. In the case of the regions that are positioned in the fingertips, the region is a matrix of 4 by 4 sensing cells. The device is connected by Ethernet connection to the computer, and the sampling rate is $850 \mathrm{~Hz}$. A client-server framework is used to publish the information given by the sensor, and to integrate it in the software system used to control the hand, based in the Robotic Operating System (ROS). Figure 1 shows the components that are used for the experiments. Kinect sensor is used in previous stages to detect the bodies in the scene, but it is not used by the algorithm and control system presented in this paper. The hand is mounted on an articulated robot Mitsubishi PA10. The robot is controlled to obtain simple trajectories that affect the state of the configuration hand-object (i.e.: lifting, dragging or squeezing).

Fig. 1. Shadow robot hand with the Tekscan Grip sensor. The Kinect sensor is used for detection of the objects.

To get reliable and understandable results, some previous steps are needed to configure the sensor. These steps are the following: balancing of regions, calibration of the system, and zeroing (see Figure 2). Each resistive cell of the regions behaves as a separate sensing unit, so the first step is to balance the response of the whole system. To do this, a uniform weight is applied to the whole sensor (complete balance), or for each region (partial balance). Each cell offers a raw output value that may vary between each of them. A balance coefficient is applied to each cell depending on its output raw value, to amplify or minimize this value.

Fig. 2. (a) Calibration curves for each of the sensor regions. (b) Map of the regions of the sensor after balancing it. White cells correspond to cells that offer higher values in the response, while black cells offer lower values.

After the sensor has been balanced, next step is to calibrate it in order to translate raw values into a real force and/or pressure value (newton, newton/centimer ${ }^{2}$ ). This process is executed in the same way as the balancing process, placing a uniform known weight over the sensor regions. In this way, a linear response between raw and real values is obtained. The final step is setting the zero value of the sensor each time it is used. Some initial or residual values may appear with no load over the sensor, which are eliminated by the zeroing of the sensor.

In regard to the Shadow hand, this device is a human-inspired robot hand with five articulated fingers and twenty degrees of freedom. The workspace of each finger and the possible movements of each joint are designed to imitate the movements of a human hand. The hand is actuated with motors, and each of the joints can be controlled both with simple PID position controllers or effort controllers. Both types of controllers are used to move the fingers during the stage of estimation of properties, switching from position control to effort control as it is going to be explained in Section 3. For the tactile servo control described in Section 5, the position controllers are used.

\section{Tactile-based In-Hand Estimation of Object's Rigidity}

In this section the proposed strategy for in-hand estimation of object's rigidity is described in detail. The proposed strategy has the goal of classifying the object in regard to the estimated rigidity value, between rigid and soft, without using a model of the object. To quantify this estimation, a percentage value, which indicates the degree of deformability produced in the object after applying external forces, is used. This value is calculated using tactile and kinematic information from the hand, so it represents the tactile sensation obtained by the robot. In this approach, a dynamic model of the object is not used nor estimated, so this exploration process can be executed for any kind of body, independently of knowing the body's mass or density. Only position and initial size of the object is assumed. The purpose of the global strategy presented in this paper is to use the obtained deformability degree to classify the objects and to adapt the contact forces depending on the estimated value. In this way, only the behavior of the object in the contact points is tracked. This approach do not depend on tracking the global deformation in the object, so a model is not needed. 
Tracking only information from the contact points instead of tracking the whole body particles using the Finite Element Method or a Mass Spring Method, has the goal of making the robotic system more agile.

It is assumed that the exploration process starts at an initial valid grasp configuration. The object size is known or previously computed using a vision system. Knowing the position and size of the object and the robot, the process of positioning the fingers in the initial contacts is a well-studied problem, in which the robot kinematics is used, and each of the possibilities of initial grasp positions is related with quality values, depending on different factors (distances to the center of the object, properties of the grasp matrix, etc.).

The strategy proposed is shown in Figure 3. The estimation stage is inspired on the human process of estimating objects' rigidity. Once an object is grasped by the fingers, the sensation of rigidity is obtained varying the contact force applied by each finger. This process mainly depends on the tactile information. To imitate this estimation using a sensorized robot hand, a "blind interaction" process has been developed.

Fig. 3. Diagram of the algorithm for object's rigidity estimation.

The process is composed by two sub-stages: position adjustment and torque readjustment. Both of them are guided by target values of the applied forces. In the position adjustment process, each fingertip is moved towards the geometric center of the object, imitating a closing movement of the hand. In the torque readjustment, torque commands are sent directly to the joints of each finger, to increase the applied force. In the first sub-stage a minimum value of contact force was set as target, and in the second, a maximum contact force value is used. The minimum threshold is a reference value that indicates that a minimum contact force exists. The maximum contact force is established as a reference force when a high pressure is applied to the object, after the maximum torque value has been applied to the fingers of the hand. After experimentation with different objects, for the robot hand and the tactile sensor that are used, the maximum contact force MAX_F value is set to 2.8 newton, which is the maximum force that the sensor detects after the maximum value of torque is applied to the joints of the fingers of the hand while it grasps a rigid body. Regarding the minimum value MIN_F, it is set to a low value of 0.3 newton, to indicate that real contact exists.

Is in the torque readjustment sub-stage where the process of estimating the object rigidity is based on. Apart from the maximum threshold, the torque readjustment is limited by the maximum possible torque to be applied to the joints of the fingers.

After the rigidity estimation stage is finished, the deformability degree value $S_{0}(\%)$ is obtained, and the object is classified into rigid or soft object taking into account the obtained value. In the presented approach, this deformability degree is obtained using the internal kinematic information of the hand and the force values of the sensors. Two ratios for each finger are used. One is a ratio between the force value (given by the tactile sensor in the fingertip region after the estimation algorithm is executed), and the maximum force value that the sensor can read (this maximum is the hard limit imposed by the mechanism of the robot hand). The second ratio relates the displacement of the finger after the estimation algorithm is executed, and the maximum displacement allowed to the finger (assuming that the object's geometry is known, the maximum corresponds with the length of the axis of the object in which the pressure is applied). Both ratios directly describe the rigidity of the object: high contact force values and low displacements indicate more rigidity, while low contact force values and high displacements indicate less rigidity. To obtain each ratio per finger the mean of this two ratios is used. The final global ratio is obtained as the mean of the values for all the fingers, in order to get a more reliable value, minimizing errors. This ratio indicates in percentage how much an object could be deformed. A value close to $0 \%$ indicates no deformation (rigidity) and a value close to $100 \%$ indicates a high deformability. Equation 1 shows how this value $\left(S_{0}\right)$ is obtained for an object, using the previously defined maximum contact force value $F_{\max }$, the displacements $d_{i}$ for each finger (obtained from hand kinematics), the final contact forces applied by each finger $f_{i}$ and the maximum distance $D_{\max }$ that fingers could move This estimation is useful for in-hand manipulation tasks, because if the obtained value is proximal to zero, a rigid body approach could be used to control a 
manipulation task, using a grasp matrix. On the other hand, for a high value of deformability, the approach for a rigid body cannot be used because the dynamics of the object is different.

$$
S_{o}(\%)=\left(\frac{\sum_{i=1}^{i=n}\left[\left(d_{i} / D_{\max }\right)+\left(\left(F_{\max }-f_{i}\right) / F_{\max }\right)\right] / 2}{n}\right) * 100
$$

\subsection{Plasticity in Objects}

Besides the deformation degree, a classification depending on the internal forces applied by the object after its deformation can be carried out to classify it between plastic or elastic objects. After the algorithm has finished, if the fingers are moved back to the initial configuration, and the current contact forces are compared with the initial ones. If the final contact forces are equal or very similar to the initial applied forces, keeping also the contact points, the object has an elastic behavior. In contrast, a loss of contacts after the hand is moved to the initial configuration, or high differences between initial and final applied forces, are a sign of a plastic behavior of the object. In the case of plastic behavior, deformations are permanent, objects do not recover their initial form, and a new grasp reconfiguration is needed.

\subsection{Isotropic and Anisotropic Bodies}

The rigidity estimation strategy may be applied both for isotropic and anisotropic bodies. For anisotropic bodies, new reconfigurations are needed to move the fingers in different axis of the object. In this way, different deformability degrees are associated to each axis of the object $(S x, S y, S z)$. Anisotropy may occur also in the same axis, in this case, the object deformability degree is a value that depends on regions of the object. Each point of contact defines a region of the object and a deformability degree in that region, which is calculated with Equation 1, but using only one finger.

\section{Maintenance of Contacts with Tactile Servo Control}

To keep the dynamic balance of the object, the contact forces applied by the fingers of the hand must counterbalance the external forces on it, in general, its weight. Moreover, the contact forces that are applied have to be inside the friction cone of the surface. Generally, weight and friction coefficient of the object are unknown, so a finger control system, independent of the weight and friction, is proposed. This control is based in the information given by the sensor regions that are positioned on the fingertips, assuming that the system hand-object is in a valid configuration where the contacts are positioned in the tips. The servo control system aims to maintain the contact force in the center of each region, and the force magnitude of each contact force is also controlled regarding to external targets, or when sliding between object and finger is detected. This control is inspired by the human behavior when an object is manipulated, and the contact forces applied vary in function of the desired deformation or in response to an unexpected movement in the object (sliding).

For in-hand manipulation tasks which involve only finger movements, the high-level task planner presented in Section 5 controls the position and contact forces applied by each finger. To control each of the fingers, a unique tactile position based controller is used for each of the fingers. The features to be tracked are the position and force magnitude of the applied force on the fingertip. The force magnitude is directly given by the tactile sensor, and the position is obtained as the position of the cell of the sensor region with the maximum value. The scheme of the controller is described in Figure 4.

Fig. 4. Control scheme of the tactile-servo controller for each finger. 
As it can be seen in Figure 4, the control variable is a vector of tactile features. The vector of features $\mathbf{f}=\left[f_{x}, f_{y}, f_{m}\right] \in \mathbb{R}^{3}$ includes the position and force magnitude of the applied force in the sensor. Position features $\left(f_{x}, f_{y}\right)$ are set as the position of the cell with the maximum values in the sensor region located at each fingertip, and the force magnitude is set as the sum of the forces of all the cells in the sensor area $A_{\text {sensor. }}$

$$
f_{m}=\sum_{i j \in R} f_{i j}
$$

The features' error $\Delta \mathbf{f}(\mathrm{t})=\left[\Delta f_{x}, \Delta f_{y}, \Delta f_{m}\right] \in \mathbb{R}^{3}$ includes the error values, respect to the target values $\mathbf{f}_{\mathrm{tgt}}=\left[f_{\_} x_{t g t}, f \_y_{t g t}, f \_m_{t g t}\right] \in \mathbb{R}^{3}$. The target in position $\left(f \_x_{t g t}, f \_y_{t g t}\right)$ is set as the central position of the sensor cell in order to maintain the contact point in the center of the sensor and fingertip. The force magnitude value $\left(f_{-} m_{\text {tgt }}\right)$ is an adaptable value given by the task planner in each iteration time. This target contact force is set depending on the task to be developed and the desired deformation on the object. Thus, this value is established according to the estimation of deformability obtained in the rigidity estimation stage, and using the reference values obtained in previous experimentation (minimum contact force value and maximum contact force value), as explained in Section 3 . For rigid objects, which have an estimated deformability degree close to $0 \%$ (not higher than 15\%), the maximum contact force value can be targeted to ensure a safe grasp in which the object is not deformed, or very slightly deformed. For soft objects, which have an estimated deformability degree above $50 \%$, low force magnitude values are targeted in order to avoid undesired deformations.

A PID controller is executed to minimize the error of the desired features $\Delta \mathbf{f}$. Each feature is controlled by a different configuration of the PID controller. The parameters of the PID that controls the error in contact force magnitude $\Delta f_{m}$ are set in order to obtain smooth responses, because an oscillation may cause undesired deformations on the object. The obtained control variable $\mathbf{u}$ is used in the next step to compute the motion of the fingers.

Once the control variable $\mathbf{u}$ is obtained, the sensor velocity $\mathbf{V}_{\mathbf{s}}{ }^{\text {sensor }} \in \mathbb{R}^{3}$ that minimizes the error in the features $\Delta \mathbf{f} \in \mathbb{R}^{3}$ is computed using a task inverted Jacobian $\mathbf{J}_{\mathbf{s}}{ }^{-1} \in \mathbb{R}^{3 \times 3}$. Only the lineal velocity components are controlled in the loop. In the fingers of the used robotic hand, the physical joints do not allow significant rotational movements of the fingertips, so the angular velocity components are not considered in the control loop. The task inverted Jacobian $\mathbf{J}_{\mathbf{s}}{ }^{-1}$ is based on the correspondence between the linear velocity components of the sensor frame, and the tactile features given by the sensor. As it can be seen in Figure 5, the axes of the plane of the sensor region $(x, y)$ match respectively the axes $X s$ and $Z s$ of the sensor frame $O_{s}$, so errors in contact force position $(x, y)$ are mapped directly into the $X_{s}$ and $Z_{s}$ axes of the sensor frame.

Mapping the contact force magnitude value into a linear velocity component in the sensor frame is not trivial. Different configurations of $\mathbf{J}_{\mathbf{s}}{ }^{-1}$ were tested to obtain an appropriate and realistic movement of the fingers, considering the coupling in the finger joints' movements. Each error step related with the contact force is related with the $Z_{s}$ and $Y_{s}$ axes of the sensor frame, in order to achieve a curved trajectory that can be followed by the fingers. The movements along this curved trajectory produce the desired variation of the contact force. In the case of the thumb, the matrix elements are negative, because the thumb is moved in the opposite direction to the rest of the fingers, so that a closing movement is produced.

The response values of the controller, given as velocity, are integrated in time to get the new position of the fingertip on each iteration. Using the kinematics of the fingers, the relative joint position for each joint is obtained, and sent to the position controllers of the real hand.

Fig. 5. (a) Visualization of the tactile sensor values in the sensor coordinates. (b) Visualization of the Shadow hand model with examples of the position of the sensor frames and palm frame.

In addition, a response to possible sliding is required, imitating the movements of the human hand, which reacts applying more pressure when sliding occurs. For this reason, the Jacobian matrix 
is modified in order to make the component $V_{y}$ of the sensor velocity dependent on the error in position $(x, y)$. Assuming that the only external force is gravity force, three different possibilities are considered:

1. The object is located above the palm: due to gravity, the object may slide in the negative direction of $Z_{s}$ axis (Error $\Delta f_{y}$ affects $V_{y}$ ).

2. The object is located below the palm: due to gravity, the object may slide in the positive direction of $Z_{s}$ axis (Error $\Delta f_{y}$ affects $V_{y}$ ).

3. The object is located at a side of the palm: due to gravity, the object may slide in the negative direction of $X_{s}$ axis (Error $\Delta f_{x}$ affects $V_{y}$ ).

The next equation shows how the error is mapped:

$$
\begin{aligned}
V_{s}^{\text {sens }} & =J_{s}^{-1} \cdot \Delta f=J_{s}^{-1} \cdot\left(\begin{array}{c}
\Delta f_{x} \\
\Delta f_{y} \\
\Delta f_{m}
\end{array}\right) \\
J_{s}^{-1} & =\left(\begin{array}{ccc}
1 & 0 & 0 \\
0 & 1 & 1 \\
0 & 1 & 1
\end{array}\right), \text { for case } 1 \\
J_{s}^{-1} & =\left(\begin{array}{ccc}
1 & 0 & 0 \\
0 & -1 & 1 \\
0 & 1 & 1
\end{array}\right), \text { for case } 2 \\
J_{s}^{-1} & =\left(\begin{array}{lll}
1 & 0 & 0 \\
1 & 0 & 1 \\
1 & 0 & 1
\end{array}\right), \text { for case } 3
\end{aligned}
$$

Considering the PID controller, the obtained velocity $\mathbf{V}_{\mathbf{s}}^{\text {sensor }}=\left[V_{x}, V_{y}, V_{z}\right] \in \mathbb{R}^{3}$ in the sensor frame is shown in the next equation:

$$
V_{s}^{\text {sens }}=J_{s}^{-1} \cdot\left(K_{p} \cdot \Delta f(t)+K_{i} \cdot \int \Delta f(t) d t+K_{d} \cdot(\Delta f(t)-\Delta f(t-1))\right)
$$

The obtained velocity in the sensor frame $O_{s}$ is transformed to the velocity $\mathbf{V}_{\mathbf{s}}^{\text {palm }} \in \mathbb{R}^{3}$ on the reference frame of each finger, placed on the palm of the hand $O_{p}$. To transform the velocity, the adjoint matrix $\mathbf{A d}_{\mathrm{T}} \in \mathbb{R}^{6 \times 6}$ is used, which is related with the current transformation matrix between $O_{p}$ and $O_{s}$ :

$$
A d_{T_{p s}}=\left(\begin{array}{cc}
R_{p s} & \hat{p}_{p s} R_{p s} \\
0 & R_{p s}
\end{array}\right)
$$

From the sensor velocity relative to the palm reference, the new position $\left(P_{s}^{\text {palm }} \in \mathbb{R}^{3}\right)$ for the fingertip is obtained integrating the value for each axis between $t$ and $t-1$. And finally, the position for each joint $(q)$ is obtained with the inverse kinematics of the hand.

\section{Task Planner}

To test the developed framework in different tasks, a high-level task planner based on basic actions is used. The robotic hand was attached to a robot arm. In this paper, only the control of the hand is considered. The movements of the arm are computed with the kinematics of the robot. The task planner, acts as a high level state machine, which controls both the target position and fingertips' contact forces at each step of a task. Each task is defined by: initial configuration, final configuration, 
and a sequence of actions to execute with the object (translation, rotation, lift, squeeze, drag). Assuming that we have the geometric description of the object without deformation, and the deformability degree has been obtained in the estimation stage, the planner changes the configurations depending on the action to be performed while the object is being held by the hand. As commented in Section 4, each finger is controlled independently in parallel by a tactile controller. Position references for the fingers have higher priority, once the position is reached the force reference is followed and is used with more priority than the position reference until a new position reference is given by the task planner. The adaptable behavior of the controller consists in changing the reference contact forces for the fingers during the execution of one task. The relationship between applied forces and deformation obtained in the estimation stage, is used by the task planner to modify the target contact forces. The average displacement $d_{a v}$ of the displacements $d_{i}$ and the average force $f_{a v}$ of all contact forces $f_{i}$ are used (see Section 3). Depending on the kind of object and the action to be performed, the target contact forces are modified in order to obtain or avoid deformations. Using the estimated values, a linear approximation in the relation between the contact force and the displacement is used to obtain the target contact force $f \_m_{\text {tgt }}$ to be applied, avoiding a deformation higher than that defined by the displacement $d_{\max }$, or considering a target deformation defined by a target displacement dtarget. The next scenarios when manipulating an object were considered, defining basic actions. The robot is moved from a trajectory start point $T_{0}$ to a trajectory end point $T_{e}$.

- Rigid objects: the maximum contact force is set on each configuration for every task step, because no deformation is produced, and higher contact forces avoid slippage; $f \_m_{\text {tgt }}=$ MAX_F.

- Soft objects:

- Translate or rotate the object while it lies on a surface (the weight of the body is not counteracted by the contact forces of the fingers); $f \_m_{\text {tgt }}=M I N \_F$.

- Lift the object increasing $f$ - $m_{\text {tgt }}$ to avoid slippage due to its weight, and initially avoiding a deformation higher than that defined by $d_{\max } ; f_{-} m_{\mathrm{tgt}}=\left(\mathrm{d}_{\max }{ }^{*} \mathrm{f}_{\mathrm{av}}\right) / \mathrm{d}_{\mathrm{av}}$.

- Drag the object exerting pressure on the surface increasing $f \_m_{\text {tgt }}$ to avoid slippage due to the friction forces, and initially avoiding a deformation higher than that defined by $d_{\text {max }} ; f_{-} m_{\text {tgt }}=\left(d_{\max }{ }^{*} f_{a v}\right) / d_{a v}$.

- Squeeze the object increasing $f \_m_{\text {tgt }}$ to produce a deformation defined by $d_{\text {target. }} f \_m_{\text {tgt }}$ $=\left(d_{\text {target }}{ }^{*} f_{\text {av }}\right) / d_{\text {av }}$.

For all the previous cases, apart from the target contact force to be obtained, the tactile servo controller reacts to possible sliding closing the fingers (increasing applied contact forces), so the target contact forces can be changed after sliding is detected.

\section{Experimentation}

Different experiments have been developed to test the algorithms presented in the previous sections. The first subsection describes experiments related with the rigidity estimation process, and the second subsection describes the experiments for the maintenance of the contacts. For these experiments, the fingers of the hand are numbered in order: index, middle, ring, little and thumb fingers are $F 1, F 2, F 3, F 4$ and $F 5$ respectively.

\subsection{Rigidity Estimation}

The rigidity estimation algorithm is carried out to obtain a value of deformation which is used to classify the object. In the experimentation, deformable objects were used. These objects maintain their shape in the space when the only force that is exerted is the gravity force. Other kind of deformable objects, such as cloth or paper, vary their shape depending on the configuration because they have an infinite number of possible deformations. This paper is focused on the study of the group of soft objects that only are deformed when external forces applied by the fingers are applied to them. We do not consider object that can be deformed by the action of gravity or robot translations. In this group of objects, it can be considered the existence of isotropic and anisotropic objects. Isotropic 
objects have a continuous and homogeneous distribution of mass and are made with the same material. Anisotropic objects have different regions, with different properties. This may be a result of different materials that compound the object, or different areas with different distribution of mass. Other possible classification takes into account the persistence of the deformation, distinguishing between temporal deformations caused to elastic objects and permanent deformations caused to plastic objects. In the first case, internal forces of the object recover its original shape, while in the second case, this internal forces are not strong enough to recover the initial shape of the object.

The rigidity estimation algorithm presented in this paper was used to estimate the deformation properties presented on the previous paragraph (stiffness, elasticity, plasticity and isotropy). In the next subsections it is shown how the estimation is used on each case. The robotic system acts autonomously, without external guidance or information given by other modules. Only targets of minimum contact force to grasp and object, and maximum contact force to apply a high pressure are given to the system, as described in Section 3. Using this tactile sensor, and after experimentation with different objects, a minimum contact force value of 0.3 newton was set and the maximum contact force value of 2.8 newton was used.

\subsubsection{Object's rigidity Estimation}

As described in Section 3, using the rigidity estimation algorithm a value that represents the sensation of rigidity is obtained, representing a value of $0 \%$ an object totally rigid, and a value close to $100 \%$ a highly deformable object. Different objects were used to test the rigidity estimation algorithm with the following results:

\section{Bottle of water}

The first object is a bottle of water made of plastic. The results of the contact forces and movements are shown in the Figure 6 . The value of deformability obtained as result is $39.10 \%$. When the test was carried out the bottle was partially filled with water. Afterwards, the test was carried out with the bottle empty of liquid, and the deformability value obtained was $45.3 \%$. When the container is empty of material, the internal forces are smaller, so the object is more deformable. For objects that have variable weight and mass distribution, such as this case of containers filled with variable quantities of liquids or other materials, a tactile sensor can help to determine these properties.

Fig. 6. (a) Evolution of the applied contact forces during the rigidity estimation of a bottle of water. (b) Displacement for each finger during this experiment.

\section{Tennis ball}

The second object used to test its rigidity is a tennis ball. The evolution of the applied contact forces and the displacements of the fingers are shown in Figure 7. The deformability degree obtained was $47.9 \%$. Two different tennis balls with different internal air pressure were tested. A second ball, with higher internal pressure was tested with an estimated value of deformability of $40 \%$. These results show that the algorithm can also be used to compare internal pressure of objects which contain air.

Fig. 7. (a) Evolution of the applied contact forces during the rigidity estimation of a tennis ball. (b)

Displacement for each finger during this experiment.

\section{Elastic Foam}

The third object for which the results of the deformability estimation are shown is an elastic foam. The evolution of the movements and applied contact forces are shown in Figure 8. For this object the estimation of deformability is $61.3 \%$. This foam was used to test the algorithm with isotropic bodies. To compare the results with different isotropic objects, and different densities, another less dense 
foam was used. The first foam has a density of 0.038 grams/centimeter ${ }^{3}$ while the second has a density of 0.015 grams/centimeter ${ }^{3}$. The algorithm gives correct results because for the first foam a value of $61.3 \%$ is obtained, while for the second a value of $69.8 \%$ is obtained, which indicates more deformability.

Fig. 8. (a) Evolution of the applied contact forces during the rigidity estimation of an elastic foam. (b) Displacement for each finger during this experiment.

\subsubsection{Plasticity Estimation}

As stated in Section 3.1., the rigidity estimation algorithm is used also to detect elastic or plastic behaviors of an object. In order to do that, fingers return to the initial grasp configuration, after the estimation has finished. Then, initial contact forces are compared with the final contact forces. If the final values are similar to the initial ones, this indicates the existence of contact with the object.

First, an elastic object is analyzed. For an elastic ball, it is shown in Figure 9 how the contact forces and the positions of the fingers vary, applying pressure to the object to deform it and then returning to the initial configuration. In this case, it can be seen that initial contact forces have values between 0.15 and 0.30 newton, and final values have values around 0.2 newton. This indicates an elastic behavior after a deformation was caused.

Fig. 9. (a) Evolution of the applied contact forces for the experiment with an elastic ball. The final contact forces are similar to the initial ones. (b) Evolution of the displacement for each finger analyzing an elastic body (elastic ball). The fingers return to the initial positions.

The second object is a plastic carton package, empty of liquid, which is deformed permanently when a pressure is applied. In Figure 10, it can be seen that after executing the algorithm for estimating the object's rigidity, and after the fingers return to the initial positions, the values of contact forces are 0 newton. This indicates a loss of contact with the object, because the deformation is maintained and the carton package does not recover the initial shape.

Fig. 10. (a) Evolution of the applied contact forces for the experiment with the carton package. The final contact forces are zero, the contact is lost. (b) Evolution of the displacement for each finger analyzing a body to detect plasticity (carton package). The fingers return to the initial positions.

\subsubsection{Isotropy and Anisotropy}

In this subsection, tests for estimating the rigidity of anisotropic objects are shown. Anisotropic objects have different distribution of mass, or different materials, which produces different deformations caused by external forces with the same force magnitude. As proposed in Section 3.2., to analyze an object with different behavior on its faces, different values of deformability are computed and associated with different faces of the object. In this example, a carton package is grasped in two different ways. In the first way the object is grasped along the object's height, using five fingers, while in the second way the object is grasped from the top and bottom faces, using only three fingers. As shown in Figure 11 and Figure 12, along each axis of the object, a different value of deformability is obtained. In the first case, the value of deformability is $57.37 \%$, and in the second case is $39.94 \%$.

Fig. 11. (a) Evolution of applied contact forces for the experiment with the carton package using the first configuration. (b) Evolution of displacements when the object is grasped with five fingers along its height. 
Fig. 12. (a) Evolution of applied contact forces for the experiment with the carton package using the second configuration. (b) Evolution of displacements when the object is grasped with three fingers using the top and bottom faces.

In Figure 13, different pictures of the experiments carried out are shown. It can be seen that, depending on the measures of each object, the hand is positioned with the palm looking down or looking to a side.

Fig. 13. (a) Experiment with the bottle of water. (b) Experiment with the ball. (c) Experiment with the elastic foam. (d) Carton package grasped along the height of the object. (e) Plasticity of a carton package. (f) Carton package grasped using top and bottom faces.

\subsection{Contact Maintenance}

In Section 4, it was described how the data obtained from the tactile sensor can be used to control the contact points between the fingers of the hand and the object. The control strategy presented uses a target contact force value for each finger. Different experiments have been developed to test the reliability of the proposed system.

In the first experiment, a deformable cube is grasped using three fingers, and the object is positioned below the hand. In this case, possible sliding would be detected in the error position related with the axis $Y$ of the sensor. Different target contact force values can be set to each finger. In this case, a target of 0.7 newton in the contact force magnitude is set to first finger and thumb, and a target contact force of 1.4 newton is set to the middle finger. In the Figure 14, the evolution of the contact forces and the error in force magnitude are shown. Using a standard configuration of the PID controller of the tactile servo with values $P=0.5, I=0.3, D=0.3$, the system is stabilized after 3.5 seconds. Besides, a response to possible sliding is needed. In the Figure 14, it can be seen that for this experiment, the error in the position $y$ component has a significant value only for the index finger. In this case, the error in position affects also the output velocities $V z$ and $V y$. In Figure 15, the output velocities for each finger are shown. No velocity $V x$ is applied to the fingers, as the position of the contact is centered in this direction of the sensor. The response of the controller applies velocities in the component $z$ and $y$, which produces a closing movement of the fingers.

Fig. 14. (a) Evolution of the applied contact forces for each finger. (b) Error in force magnitude. (c) Error in position, using the tactile servo controller.

Fig. 15. Output velocities for each finger in contact with the object when the contacts are controlled by the tactile servo controller. (a) Thumb. (b) First finger. (c) Middle finger.

In the second experiment, different target values are used for each of the fingers. Each tactileservo controller acts independently. In the Figure 16, the evolution of the applied contact forces, and the error in force magnitude and position are shown. While the error in position is very low, the error in force magnitude is minimized with the output velocities shown in Figure 17. The system is stabilized after 2.5 seconds.

Fig. 16. (a) Evolution of the applied contact forces for each finger. (b) Error in force magnitude. (c) Error in position, using the tactile servo controller.

Fig. 17. Output velocities for each finger in contact with the object when the contacts are controlled by the tactile servo controller. (a) Thumb. (b) First finger. (c) Middle finger. (d) Ring finger. 


\subsection{Task planner}

In this section an example of a manipulation task is shown to illustrate how the task planner works. The task is composed of different actions, in which different target contact forces are set. The object that is used is a soft elastic sponge. The example task consist in moving the object without lifting it, drag the object applying force into the surface, lift the object, and squeeze it. For this object, a deformability degree of $62 \%$ was obtained, with an average contact force value of 0.9 newton and an average displacement value of 0.035 meter. With these values, and focusing in the adaptation of the contact forces, the resulting target contact forces for each action are shown in Table 1:

Table 1: The table shows the target contact forces that are set as reference for the tactile-servo controllers, depending on the action to be performed and the features extracted from the object.

\begin{tabular}{|c|c|c|}
\hline Action & Force Computation & Target Contact Force \\
\hline Move the object & $\mathrm{F}_{-} \mathrm{m}_{\mathrm{tgt}}=\mathrm{MIN} \mathrm{F}$ & $0.25 \mathrm{~N}$ \\
\hline $\begin{array}{c}\text { Drag the object with max. } \\
\text { deformation }=0.02 \mathrm{~m}\end{array}$ & $\mathrm{~F}_{-} \mathrm{m}_{\mathrm{tgt}}=\left(\mathrm{d}_{\mathrm{max}} * \mathrm{f}_{\mathrm{av}}\right) / \mathrm{d}_{\mathrm{av}}$ & $0.51 \mathrm{~N}$ \\
\hline $\begin{array}{c}\text { Lift the object with max } \\
\text { deformation }=0.01 \mathrm{~m}\end{array}$ & $\mathrm{~F}_{-} \mathrm{m}_{\mathrm{tgt}}=\left(\mathrm{d}_{\max } * \mathrm{f}_{\mathrm{av}}\right) / \mathrm{d}_{\mathrm{av}}$ & $0.38 \mathrm{~N}$ \\
\hline $\begin{array}{c}\text { Squeeze the object with target } \\
\text { deformation }=0.04 \mathrm{~m}\end{array}$ & $\mathrm{~F}_{-} \mathrm{m}_{\mathrm{tgt}}=\left(\mathrm{d}_{\mathrm{max}} * \mathrm{f}_{\mathrm{av}}\right) / \mathrm{d}_{\mathrm{av}}$ & $1.02 \mathrm{~N}$ \\
\hline
\end{tabular}

\section{Conclusions}

In this paper, a new approach for in-hand manipulation tasks with robot hands is presented. The main contribution of this work is the versatility to manipulate different kind of objects, both rigid and deformable. For future robots, the possibility of interacting with any kind of objects, in any environment, is a key feature. The lack of adaptation to different objects, makes the robot system less useful.

The presented approach has been tested in a laboratory with a set of objects that a robot could have to recognize and manipulate. From the experiments shown, it is demonstrated the reliability of this approach, both for estimating objects' rigidity and for keeping contact points at a specific configuration. How much an object can be deformed, defines the contact forces that a robot hand could apply. The control of the contact points is basic to carry out manipulation tasks. Both goals are accomplished with the strategies presented here. As future work, the estimation may be adapted to recognize more complex deformable objects, such as cloth, whose deformations are more complex. In this cases, support from a vision system would be needed.

The complexity of the dynamics of soft bodies makes necessary to develop complex control strategies to move and deform an object in a controllable way. The estimation stage and the servotactile control will be used as the basis of a hand controller adaptable to different objects. The control of the manipulation is based on the dynamics of the hand and object, so, if the dynamics of the object is not known, a previous estimation stage to obtain this dynamic properties is needed.

\section{References}

1. Yoshikawa, T. Multifingered robot hands: Control for grasping and manipulation. Annual Reviews in Control vol. 34 (2) p. 199-208. 2010.

2. Huang J.L., Clement R., Sun Z.H., Wang J., Zhang W.J. Global Stiffness and Natural Frequency Analysis of Compliant Mechanisms with Embedded Actuators with a General-Purpose Finite Element System. International Journal of Advanced Manufacturing Technology. 2012. DOI 10.1007/s00170-012-4243-8

3. Khalil, F.F.; Payeur, P. Dexterous Robotic Manipulation of Deformable Objects with Multi-Sensory Feedback - a Review. Robot Manipulators Trends and Development, Agustin Jimenez and Basil M Al Hadithi (Ed.), ISBN: 978-953-307-073-5, InTech, DOI: 10.5772/9183. 2010. 
4. Luo, Y.; Nelson, B.J. Fusing force and vision feedback for manipulating deformable objects. Journal of Robotic Systems, vol. 18(3), PP. 103-117. 2001.

5. Bimbo, J.; Seneviratne, L.; Althoefer, K.; Liu, H. Combining touch and vision for the estimation of and object's pose during manipulation. In Proc. IEEE/RSJ Int. Conf. on Intelligent Robots and Systems (IROS), Tokyo, 2013, pp. 4021-4026. 2013.

6. Khalil, F. F.; Payeur, P.; Cretu, A-M. Integrated Multisensory Robotic Hand System for Deformable Object Manipulation. Proc. Int. Conf. Robotics and Applications, pp. 159-166, Cambridge, Massachusetts, US, Nov. 2010. 2010.

7. Li, Q.; Elbrechter, C.; Haschke, R.; Ritter, H. Integrating vision, haptics and proprioception into a feedback controller for in-hand manipulation of unknown objects. Proc. IEEE/RSJ Int. Conf. on Intelligent Robots and Systems (IROS), Tokyo, 2013, pp. 2466-2471. 2013.

8. Khalil, F.F.; Curtis, P.; Payeur, P. Visual monitoring of surface deformations on objects manipulated with a robotic hand. Robotic and Sensors Environments (ROSE). In Proc. 2010 IEEE International Workshop, on vol., no., pp.1, 6, 15-16 Oct. 2010doi: 10.1109/ROSE.2010.5675327. 2010.

9. Corrales, J.A.; Torres, F.; Perdereau, V. Finger Readjustment Algorithm for Object Manipulation Based on Tactile Information. Int J Adv Robot Syst, 2013, 10:9. DOI: 10 5772/53561. 2013.

10. Kien-Cuong, N.; Perdereau, V. Fingertip force control based on max torque adjustment for dexterous manipulation of an anthropomorphic hand. Intelligent Robots and Systems (IROS), 2013 IEEE/RSJ International Conference on vol., no., pp.3557-3563, 3-7 Nov. 2013. 2013.

11. Han, L.; Trinkle, J. Dexterous Manipulation by Rolling and Finger Gaiting. In IEEE International Conference on Robotics and Automation, Leuven, Belgium, 1998; pp. 730-735. 1998.

12. Romano, J. M.; Hsiao, K.; Niemeyer, G.; Chitta, S.; Kuchenbecker, K. J. Human-inspired robotic grasp control with tactile sensing. IEEE Transactions on Robotics. http://doi.org/10.1109/TRO.2011.2162271. 2011.

13. Cutkosky, M. R.; Hyde, J. M. Manipulation Control with Dynamic Tactile Sensing. In International Symposium on Robotics Research. 1993.

14. Dang, H.; Weisz, J.; Allen, P. K. Blind grasping: Stable robotic grasping using tactile feedback and hand kinematics. In Proceedings - IEEE International Conference on Robotics and Automation. http://doi.org/10.1109/ICRA.2011.5979679. 2011.

15. Howe, R. D. Tactile Sensing and Control of Robotic Manipulation. Journal of Advanced Robotics, 8(3), 245261. 1994.

16. Yousef, H.; Boukallel, M.; Althoefer, K. Tactile sensing for dexterous in-hand manipulation in robotics - A review. Sensors and Actuators A: Physical vol. 167 (2), p. 171-187. 2011.

17. Wettels, N.; Loeb, G. E. Haptic feature extraction from a biomimetic tactile sensor: Force, contact location and curvature. In 2011 IEEE International Conference on Robotics and Biomimetics, ROBIO 2011. http://doi.org/10.1109/ROBIO.2011.6181676. 2011.

18. Liu, H.; Song, X.; Nanayakkara, T.; Seneviratne, L. D.; Althoefer, K. A computationally fast algorithm for local contact shape and pose classification using a tactile array sensor. In Proceedings - IEEE International Conference on Robotics and Automation (pp. 1410-1415). 2012.

19. Chitta, S.; Sturm, J.; Piccoli, M.; Burgard, W. Tactile sensing for mobile manipulation. IEEE Transactions on Robotics, 27(3), 558-568. 2011.

20. Li, Q.; Schürmann, C.; Haschke, R.; Ritter, H. A control framework for tactile servoing. Proceedings of Robotics: Science and Systems. 2013.

21. Shadow Robot, "Dexterous Hand". [Online]. Available: http://www.shadowrobot.com/products/dexteroushand/.

22. Tactile Sensor Tekscan. [Online]. Available: http://www.tekscan.com/grip-pressure-measurement. 